\title{
About NAET; (Nambudripads Allergy Elimination Technique) A Revolutionary Technique for Allergies and More!
}

\begin{abstract}
About NAET
NAET is a unique method to balance energy blockages to treat allergies and all manner of ailments. NAET works on the basis that anyone can be allergic to any substance, be it chemical or natural and these allergies can cause illness. In successfully treating a patient, special diets don't have to be adhered to for long periods, the items treated need avoiding for 25 hours after treatment, sometimes slightly longer. NAET combines western science, kinesiology, chiropractic, acupuncture and nutritional therapies.
\end{abstract}

\section{Advantages}

a. A key advantage is that NAET is non-invasive, without unpleasant blood or skin tests, injections or medication. The aim is to find the food and environmentally sensitive substances which trigger a reaction and to target these with pain free and harmless treatments.

b. Therefore, NAET is suitable for all ages.

c. NAET has proved its effectiveness over the course of many years. Patients come with hay fever and dairy intolerance to neurodermatitis, migraines and hyperactivity. Results are the proof for how well NAET works.

d. NAET is much more structured, detailed and comprehensive.

e. All NAET instructors must be certified by Devi Nambudripad in the US, to ensure that a high standard permeates throughout.

\section{Allopathy \& Western Sciences}

Knowledge of the brain, cranial nerves, spinal nerves and autonomic nervous system from Western medicine enlightens us about the body's efficient multilevel communication network. Through this network of nerves, vital energy circulates through the body carrying negative and positive messages from the brain to each and every cell in the body and back to the brain.

Knowledge of the nervous system, its origin, travel pathway, the organs and tissues that will benefit from its nerve energy supplies (target organs and tissues) and its destination helps us to understand the energy distribution of particular spinal nerves that emerge from the 31 pairs of spinal nerve roots.

\section{Kinesiology}

Kinesiology is the art and science of movement of the human body. Kinesiology is used in NAET to compare the strength and weakness of any muscle (also known as neuromuscular sensitivity testing (NST)) of the body in the presence and absence of any substance.

Volume 8 Issue 3 - 2017
Helen Bowminion
Holistic healthcare, Clinics in Yorkshire \& Cambridge, UK
*Corresponding author: Helen Bowman, Holistic
healthcare, Clinics in Yorkshire \& Cambridge, UK,
Email: helen@holistichealthcare.eu
Received: February 03, 2017 | Published: August 02, 2017

A measured weakness in the presence of a substance is due to the effect of an allergy to the item the person is touching. This simple method can be used to detect your allergens.

\section{Chiropractic}

Chiropractic technique helps us to detect the nerve energy blockage in a specific nerve energy pathway by detecting and isolating the exact nerve root being pinched. The exact vertebral level in relation to the pinched spinal nerve root helps us to trace the travel route, the destination and the target organs of that particular energy pathway.

D. D. Palmer, considered the "Father of Chiropractic," said, "too much or too little energy is disease." A pinched nerve can cause disturbance in the energy flow.

Chiropractic medicine postulates that a pinched nerve or any such disturbance in the energy flow can cause disease, revealing the importance of maintaining an uninterrupted flow of nerve energy. A pinched nerve or an obstruction in the energy flow is often the result of an allergy. Spinal manipulation at the specific vertebral level of the pinched nerve, can relieve the obstruction of the energy flow and help the body to arrive at a state of homeostasis.

\section{Acupuncture/Oriental Medicine}

Yin-Yang theory from Oriental medical principles teaches the importance of maintaining homeostasis in the body. According to Oriental medical principles, "when the body is in perfect balance, no disease is possible." Any disturbance in the homeostasis can cause disease. Any allergen capable of producing a weakening muscular effect in the body can cause disturbance in homeostasis. Hence, diseases can be prevented and cured by maintaining homeostasis. According to acupuncture theory, acupuncture and/or Acupressure at certain acupuncture points is capable 
of bringing the body to a state of homeostasis by removing the energy blockages from the energy pathways known as meridians.

When the blockages are removed, energy can flow freely through the energy meridians, thus bringing the body in perfect balance.

\section{Nutrition}

You are what you eat! The secret to good health is achieved through right nutrition. What is right nutrition? And how do you get it? When you can eat nutritious foods without discomfort and assimilate their nutrients, that food is said to be the right food. When you get indigestion, bloating, or other digestive troubles upon eating the food, that food is not helping you function normally. This is due to an allergy to the food. Different foods react differently in different people. What is food for some may be poison for others. You've probably heard the expression, "one man's meat is another man's poison." So, it is very important to clear the allergy to the nutrients. Allergic people can tolerate food that is low in nutrition better than nutritious food. But upon clearing the allergy, you should try to eat more wholesome, nutritious foods. You should avoid refined, bleached food that is devoid of nutrients.

Many people, who are feeling poorly due to undiagnosed food allergies, may take vitamins or other supplements to increase their vitality after they get treated for the specific allergy. If they happen to be allergic to the nutritional supplements they are taking, this can make them feel worse. Only after clearing those allergies, can their bodies properly assimilate them. Therefore, nutritional assessment should be done periodically and if needed, appropriate supplements should be taken to receive faster results. 